\title{
Flies come through again, period
}

\author{
Amita Sehgal \\ Chronobiology Program, Howard Hughes Medical Institute (HHMI), Perelman School of Medicine, University of Pennsylvania, Philadelphia, Pennsylvania, USA.
}

effrey Hall, Michael Rosbash, and Michael Young have been awarded the 2017 Nobel Prize in Physiology or Medicine for elucidating the molecular mechanism of the circadian ( 24-hour) clock. Starting with the cloning of the period (per) gene, these investigators proceeded to demonstrate how endogenous clocks are composed of transcription-translation feedback loops (TTFLs) that drive rhythms in much of physiology. Beyond their fundamental significance for understanding normal physiology, the identification of clock mechanisms has led to a deeper appreciation of the health consequences of circadian disruption. Importantly, the Nobel prize-winning discoveries were made using a Drosophila melanogaster model, underscoring yet again the power of the fruit fly for biomedical research.

Most physiological processes and behaviors display changes over a 24-hour period in synchrony with the day-night cycle. Sleep, body temperature, metabolic activity, and secretion of many hormones, such as cortisol and insulin, represent but a few of the processes that are different in the morning versus the evening. These rhythms are driven by endogenous clocks that have a periodicity of approximately 24 hours, hence the name "circadian" from "circa" (about) and "dies" (day). Circadian rhythms are clearly an integral part of physiology, and disruptions in them are associated with a myriad of disorders, making it important to understand the mechanisms that generate them.

\section{A genetic basis for circadian clocks}

Although daily rhythms that persist in the absence of light-dark cycles had been noted in the 1700s, the idea that they are generated by endogenous clocks was not accepted until the 20th century. The circadian period varies from species to species and, as noted above, usually does not precisely match the periodicity of the environment, providing support for an internal rhythm-generating process. A genetic basis for clocks was demonstrated by Bunning, whose work with bean plants in the 1930s demonstrated that circadian period is heritable. Subsequently, a historic screen for relevant genetic factors was conducted by Ron Konopka, a graduate student in the laboratory of Seymour Benzer at Caltech. Konopka mutagenized fruit flies (Drosophila melanogaster) and assayed for $\mathrm{X}$ chromosome mutations that altered circadian rhythms. He succeeded in identifying a fly line that had a short period $(\sim 19$ hours), a second with a long period ( 29 hours), and yet another that lacked rhythms altogether. These mutations mapped to the same gene, which Konopka termed the period (per) gene (1).

Isolation of the per gene awaited the efforts of the trio that was recognized this fall. Using newly developed molecular tools in the early 1980s, Jeffrey Hall and Michael Rosbash of Brandeis University and Michael Young of Rockefeller University began the search for the per gene. Both groups localized per to a small region on the $\mathrm{X}$ chromosome, and following extensive analysis of candidate transcripts, they succeeded in pinpointing the relevant gene (2, 3). Initial analysis of per did not provide reliable answers as to how it might be involved in timekeeping, but its isolation was nevertheless an enormous breakthrough, not only from a circadian point of view, but also from a behavioral biology perspective. Phenotypes of the per mutants were found to result from point mutations that led to amino acid substitutions in the case of the short and long alleles and to a nonsense codon that truncated the protein in the arrhythmic allele. Thus, per became a paradigm for a behavioral gene, one in which even a single nucleotide change could profoundly and specifically affect behavior.

\section{A mechanistic understanding of the clock}

The first insights into the clock mechanism came from the finding, in the Hall and Rosbash laboratories, that per protein (PER) and mRNA are expressed cyclically (Figure 1). The surprise was that cycling of the mRNA appeared to depend on the protein, such that it was altered or lost in the per missense or null mutants, respectively, and rescue by functional PER could even drive cycling of the nonsense mutationcontaining per $^{01}$ transcript (4). This observation led to the model that per functions in a feedback loop that could be an integral part of the clock. Indeed, discovery of the second circadian clock gene timeless (tim) in the Young laboratory (5) substantiated the feedback loop mechanism and provided the basis for a clock model in which the per and tim genes are coregulated and cyclically expressed, and the two proteins negatively regulate transcription of both mRNAs. PER and TIM interact directly and display mutual regulation, most notably in ensuring nuclear expression of the heterodimer. Within the nucleus, they negatively regulate their own expression by inhibiting the activity of their transcriptional activators CLOCK (CLK) and CYCLE (CYC), discovered subsequently by the Hall and Rosbash groups. Timely expression and activity of PER and TIM are critical for the appropriate regulation of transcription and also depend on controlled phosphorylation events. The Rosbash laboratory showed that clock proteins are phosphorylated rhythmically, and the Young laboratory identified clock kinases, the first and best known being casein kinase $1 \varepsilon$, which they termed doubletime $(d b t)$ and which phosphorylates and destabilizes PER. In fact, TIM counteracts the effect of DBT to stabilize PER. Synchrony of the clock with light, a key feature of circadian clocks, is also accounted for by this clock mechanism, specifically by lightmediated degradation of the TIM protein that consequently affects PER and the entire loop (reviewed in ref. 6). 


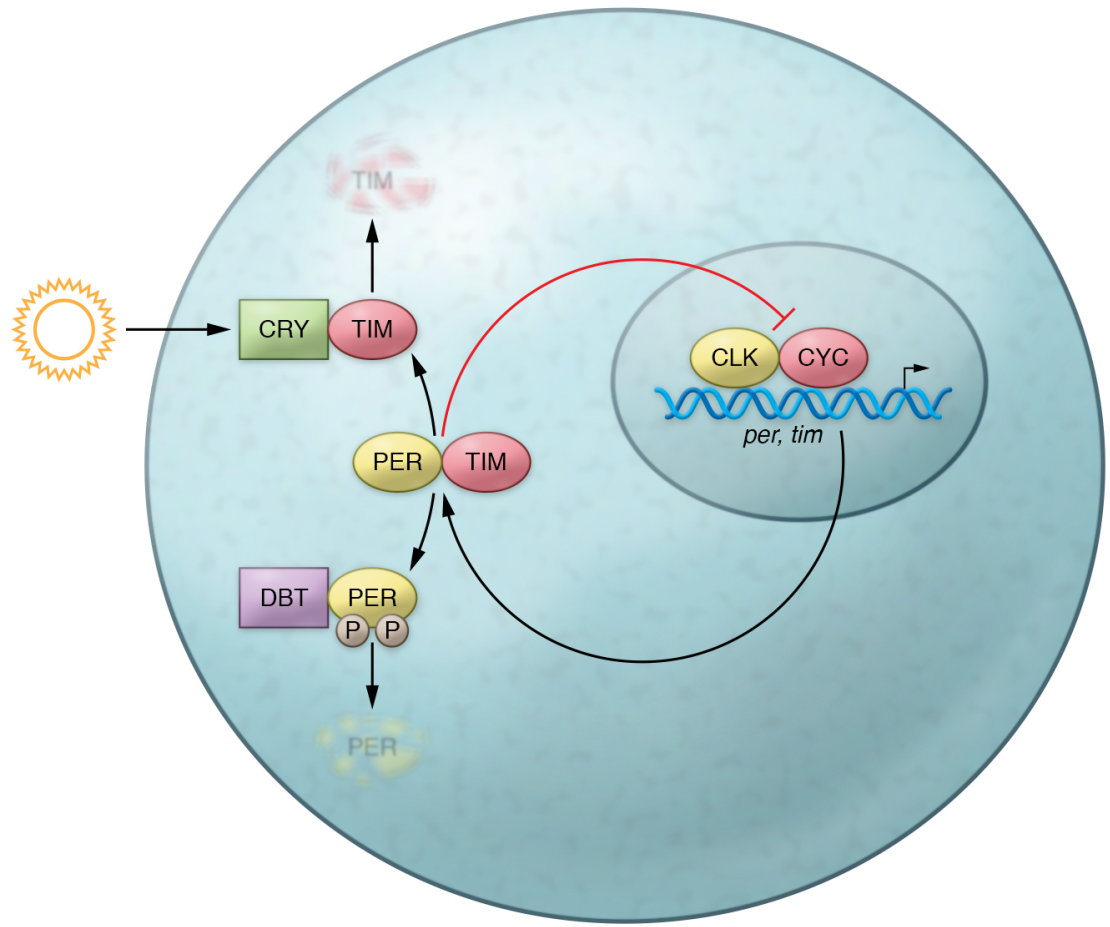

Importantly, a negative feedback loop consisting of two coregulated genes is now the prevailing model for all animal clocks, and most of the molecular components are also conserved from flies to mammals. In fact, the Clock gene was identified first in mice by the laboratory of Joseph Takahashi (7), and in the past two decades molecular circadian research in Drosophila and mammals has moved in parallel. The significance of the fly work cannot, however, be overstated. The molecular lesion in the first characterized human circadian disorder was traced to the Per2 gene (mammals have 3 period genes), specifically to phosphorylation of PER2 by casein kinase $1 \varepsilon(8)$, and subsequent human studies have also been informed by clock mechanisms identified in Drosophila.

\section{Conclusions}

Our current understanding of clocks now involves many added layers of complexity, including additional connected loops, but the basic principle described above can explain circadian regulation in general. Transcriptional effects of clock proteins are not restricted to their own mRNAs, but apply to many genes, thereby driving rhythmic expression of those genes. The finding, again first made in flies by the prize winners, that clock genes are expressed not only in the brain, but also in other organs, has led to the concept of a circadian system that consists of multiple synchronized clocks across the organism. In any given organ, as many as $15 \%$ to $20 \%$ of the transcripts may cycle under clock control, and they are typically specific for the functional output of that organ. Loss of synchrony among body clocks, or of endogenous clocks to the environment, has deleterious physiological consequences. Given that circadian desynchrony/disruption - caused by jet lag, shift work, constant exposure to light, or even mistimed feeding - is quite common in modern society, it could be a significant contributor to pathological conditions, which is supported by findings of circadian abnormalities during aging and in metabolic, cardiovascular, neurological, neuropsychiatric, and other diseases. Recognition of the circadian field by the Nobel Assembly at Karolinska Institute is timely and will undoubtedly focus attention on these daily cycles and their importance for health and disease pathogenesis.

\section{Acknowledgments}

Work in the laboratory is supported by NIH grant R37NS048471.
Figure 1. The molecular clock in Drosophila. In the major feedback loop of the Drosophila clock, the PER and TIM proteins inhibit activity of their transcriptional activators CLK and CYC at a specific time of day. Cycles of PER and TIM expression and activity are maintained through rhythmic transcription as well as through timely phosphorylation, degradation, and nuclear expression of the two proteins. Phosphorylation of PER by DBT (CK1ع) destabilizes it, an effect that is countered by TIM expression in the early night. Entrainment of the loop to light involves degradation of the TIM protein in response to signals transmitted by the cryptochrome (CRY) photoreceptor. In addition, the visual system can also entrain the clock. The figure is focused on the early findings that led to the awarding of this prize and does not include additional components, including interlocked loops, that are now known.
Address correspondence to: Amita Sehgal, 10-136 Smilow Research Center, 3400 Civic Center Blvd., Bldg. 421, Philadelphia, Pennsylvania 19104-5158, USA. Phone: 215.573.2985; Email: amita@ pennmedicine.upenn.edu.

1. Konopka RJ, Benzer S. Clock mutants of Drosophila melanogaster. Proc Natl Acad Sci U S A. 1971;68(9):2112-2116.

2. Zehring WA, et al. P-element transformation with period locus DNA restores rhythmicity to mutant, arrhythmic Drosophila melanogaster. Cell. 1984;39(2 pt 1):369-376.

3. Bargiello TA, Jackson FR, Young MW. Restoration of circadian behavioural rhythms by gene transfer in Drosophila. Nature. 1984;312(5996):752-754.

4. Hardin PE, Hall JC, Rosbash M. Feedback of the Drosophila period gene product on circadian cycling of its messenger RNA levels. Nature. 1990;343(6258):536-540.

5. Sehgal A, Price JL, Man B, Young MW. Loss of circadian behavioral rhythms and per RNA oscillations in the Drosophila mutant timeless. Science. 1994;263(5153):1603-1606.

6. Dubowy C, Sehgal A. Circadian rhythms and sleep in Drosophila melanogaster. Genetics. 2017;205(4):1373-1397.

7. Vitaterna $\mathrm{MH}$, et al. Mutagenesis and mapping of a mouse gene, Clock, essential for circadian behavior. Science. 1994;264(5159):719-725.

8. Toh KL, et al. An hPer2 phosphorylation site mutation in familial advanced sleep phase syndrome. Science. 2001;291(5506):1040-1043. 